\title{
ИССЛЕДОВАНИЕ ПРОЦЕССОВ РЕЛАКСАЦИИ В НИЗКОТЕМПЕРАТУРНЫХ ПРИМЕСНЫХ СИСТЕМАХ МЕТОДОМ РАССЕЯНИЯ НА СВЕТОНАВЕДЕННЫХ СПЕКТРАЛЬНЫХ PЕШЕТКАХ
}

\author{
(Представил П. Саари)
}

\section{Введение}

Метод фотовыжигания устойчивых спектральных провалов $\left[{ }^{1-4}\right]$ является мощным инструментом исследования процессов распада фотовозбуждения примесных молекул в твердых низкотемпературных матрицах, имеющих широкие неоднородно уширенные спектры поглощения и испускания. Однако продолжительность цикла выжигания и регистрации провала составляет обычно как минимум $1-100$ с, и тем самым определяемые по ширине провала однородные спектральные характеристики и соответствующие времена релаксации возбужденного состояния молекулы могут быть искажены медленными флюктуациями частоты перехода за цикл измерения. Для установления и исключения таких искажений следует провести измерение времен релаксации за более короткий период.

В данной работе показано, что весьма перспективным является метод рассеяния на светонаведенных спектральных решетках (РССР) с использованием наносекундных некогерентных лазерных импульсов с широкими спектрами генерации, который применялся для определения возможного вклада спектральной диффузии в релаксационные характеристики примесных молекул в стеклообразных матрицах, определенных по результатам фотовыжигания спектральных провалов.

Указанный метод назывался раньше методом аккумулированного фотонного эха с некогерентным светом $\left[{ }^{5-8}\right]$ или методом четырехволнового смешивания с временной задержкой $\left[{ }^{9-11}\right]$. Предложенное нами название подчеркивает сходство метода РССР с хорошо известным методом рассеяния на светонаведенных пространственных решетках и на его связь с пространственно-временной голографией [ $\left.{ }^{12-14}\right]$. Как показано в $\left[{ }^{13}\right]$, характерные особенности пространственно-временных голограмм остаются в силе и в случае некогерентных световых полей. Это дает возможность при трактовке РССР опираться на общие и физически наглядные представления, выработанные в пространственновременной голографии.

В данной работе:

1) исследуются возможности применения метода РССР в условиях насыщения поглощения;

2) на основе результатов по пространственно-временной голографии $\left.{ }^{13-15}\right]$ интерпретированы кривые затухания интенсивности сигнала РССР в низкотемпературных примесных системах; 
3) измерены времена фазовой релаксации $T_{2}$ системы октаэтилпорфин-полистирол при температурах $(2,15-1,60 \mathrm{~K})$ и проведено сравнение с результатами, полученными из экспериментов по стационарному выжиганию провалов.

\section{Описание и анализ особенностей метода рассеяния на светонаведенных спектральных решетках}

Метод РССР заключается в следующем. Лазерный импульс разделяется на два импульса, которые направляются на объект под малым углом $\theta$ и с задержкой $t_{12}$ относительно друг друга. Если однородная ширина линий молекулярной системы $\Gamma_{00} \ll 1 / t_{12}$, то такая пара импульсов создает в неоднородно уширенном спектре примесного поглощения синусоидальную решетку пропускания с шагом $\Delta v=1 / t_{12}$. Эта решетка появляется в результате селективного уменьшения заселенности основного уровня согласно фурье-спектру поглощенных импульсов и сохраняется до тех пор, пока не проходит энергетическая релаксация фотовозбуждения и не восстанавливается первоначальная заселенность основного состояния.

В результате рассеяния третьего, зондирующего импульса на этой частотной светонаведенной решетке с задержкой $t_{34}=t_{12}$ высвечивается четвертый сигнальный импульс.

По своему характеру сигнальный импульс есть когерентный отклик среды типа стимулированного светового эха $\left[{ }^{16,17}\right]$. Относительная интенсивность сигнала зависит от глубины модуляции наведенной решетки, которая определяется сверткой фурье-спектра возбуждающих импульсов и однородного спектра поглощения примеси $K\left(\omega-\omega_{0}\right)$. Уменьшение шага $\Delta v\left(\right.$ рост $\left.t_{12}\right)$ и его становление сравнимым с $\Gamma_{00}$ приводят к увеличению сглаживающего действия однородного спектра $K\left(\omega-\omega_{n}\right)$ в свертке и соответственно к уменьшению контраста и сигнала. Если форма однородного спектра лоренцова, интенсивность сигнала падает при увеличении $t_{12}$ экспоненциально $\left[{ }^{6}\right]$

$$
I\left(t_{12}\right) \sim \exp \left(-4 t_{12} / T_{2}\right) .
$$

Таким образом, по зависимости интенсивности сигнала РССР от задержки $t_{12}$ между возбуждающими решетку импульсами можно определить время фазовой релаксации $T_{2}$. При этом временное разрешение определяется не длительностью, а временем когерентности импульсов $\tau_{c}\left[{ }^{5,9,18}\right]$. Следовательно, при помощи наносекундных лазерных импульсов можно провести измерение субнаносекундных и даже субпикосекундных времен $T_{2}$, если только спектральная ширина импульсов $\Delta v \sim 1 / \tau_{c}$ достаточно большая. С изменением плавно задержки $t_{12}$ эффективное время определения каждой точки на кривой (1) может достигать $10-20$ нс. Если теперь до прихода следующей тройки импульсов восстанавливается полностью первоначальное состояние образца, исключается влияние более медленных процессов на результат измерения.

Удобным с точки зрения эксперимента свойством РССР является хорошая направленность сигнала согласно условию фазового синхронизма

$$
\vec{k}_{4}=\vec{k}_{3}+\vec{k}_{2}-\vec{k}_{1}
$$

где $\vec{k}_{n}(n=1,2,3,4)$ волновые векторы пары возбуждающих решетку импульсов, зондирующего и эхо-импульсов соответственно. 
Из формулы (2) следует, что если меняется очередность возбуждающих импульсов $\left(\vec{k}_{1} \rightleftarrows \vec{k}_{2}\right)$, то направление импульса отклика $\left(\vec{k}_{4}\right)$ переключается в другую сторону от направления зондирующего импульса (рис. 1).

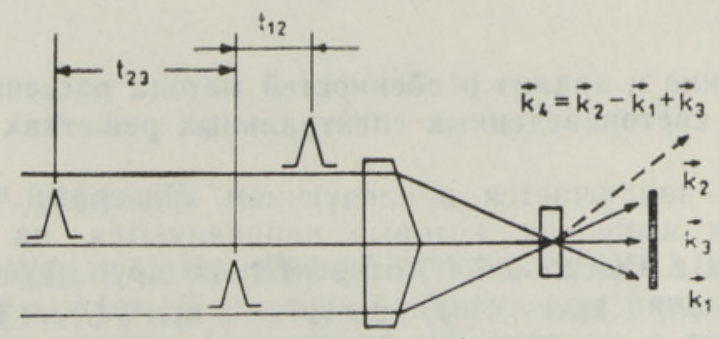

Рис. 1. Геометрия импульсов возбуждения. Направление сигнала РССР $\vec{k}_{4}$ зависит от очередности поступления импульсов $\vec{k}_{1}$ и $\vec{k}_{2}$ на образец.

Временные и пространственные характеристики сигнала РССР могут быть объяснены в рамках теории стимулированного светового эха $\left[{ }^{17}\right]$. В данной работе мы постараемся описать поведение сигнала РССР исходя из структуры и свойств индуцированной пространственноспектральной решетки как линейного фильтра $\left[{ }^{13,}{ }^{14}\right]$. Под спектральными решетками мы везде подразумеваем решетки, индуцированные по неколлинеарной схеме, описанной на рис. 1, которые обеспечивают угловую селективность рассеяния.

1. Как показано в [ $\left.{ }^{12-14}\right]$, задержка сигнального импульса относительно третьего зондирующего импульса появляется за счет того, что при прохождении зондирующего импульса через образец как линейный фильтр, его спектр преобразуется согласно спектру пропускания индуцированной в образце спектральной решетки. Как уже отмечалось, шаг этой решетки опрсделяется задержкой $t_{12}$ между возбуждающими решетку первыми двумя импульсами. В итоге, согласно фурье-образу спектра выходного излучения, за зондирующим импульсом с задержкой $t_{34}=t_{12}$ высвечивается рассеянный решеткой импульс.

2. Направленность сигнала обусловлена дифракцией монохроматических фурье-компонентов зондирующего импульса на скрытой пространственной структуре индуцированных спектральных решеток. Пространственная структура спектральных решеток скрыта в том смысле, что в условиях образования спектральной решетки $\left(t_{12}>\tau_{\mathrm{c}}\right)$ возбуждающие импульсы не могут интерферировать непосредственно и не формируют в образце пространственную голографическую решетку, наблюдаемую в «белом» свете. Однако анализ показывает, что регулярные пространственные решетки в образце все же существуют для всех фурье-компонентов зондирующего импульса в отдельности. Но начальные фазы этих «монохроматических подрешеток» со слегка различными постоянными решетки раздвинуты таким образом, что интегрально по спектру зондирующего импульса пропускание образца в каждой точке пространства остается одинаковой. В спектральной решетке наблюдается лишь зависимость фазы модуляции от пространственной координаты. В итоге, фурье-компоненты зондирующего импульса дифрагируют на резонансных «монохроматических подрешетках», согласно условию фазового синхронизма (2), хорошо известного из теории дифракционных решеток. 
3. Односторонность и переключение направления рассеяния обеспечиваются вкладом в рассеяние фазовой составляющей спектральной решетки, возникающей за счет модуляции показателя преломления среды $\left[{ }^{13}\right]$. Согласно соотношению Крамерса-Кронига, последняя неизбежно сопровождает спектральную модуляцию амплитудного пропускания.

Учет только амплитудной составляющей спектральной решетки приводил бы к выводу о двустороннем рассеянии в направлениях $\vec{k}_{4}=\vec{k}_{3} \pm\left(\vec{k}_{2}-\vec{k}_{1}\right)$, как и в случае пространственных индуцированных решеток. Однако, если один из компонентов рассеяния (в направлении $\left.\overrightarrow{k_{3}}+\left(\vec{k}_{2}-\vec{k}_{1}\right)\right)$ высвечивается с задержкой $t_{12}$, то другой компонент (в направлении $\left.\vec{k}_{3}-\left(\vec{k}_{2}-\vec{k}_{1}\right)\right)$ должен появиться с опережением на $t_{12}$ относительно зондирующего импульса, что не удовлетворяет принципу причинности линейных откликов.

Учет при РССР модуляции как мнимой, так и действительной части диэлектрической проницаемости среды, т. е. учет сопряженности амплитудной и фазовой спектральных решеток устраняет это противоречие и автоматически обеспечивает соблюдение причинности $\left[{ }^{13,14}\right]$, налагая запрет на рассеяние в направлении $\vec{k}_{4}=\vec{k}_{3}-\left(\vec{k}_{2}-\vec{k}_{1}\right)$.

Исходя из односторонности рассеяния, переключение направлений рассеяния при изменении очередности возЈуждающих решетку импульсов становится очевидным.

В заключение следует еще обратить внимание на некоторые вопросы, немаловажные с точки зрения разработки экспериментальной схемы.

Во-первых, в ходе наведения спектральных решеток наблюдается самодифракция, как и в случае наведенных пространственных голографических решеток, которая, однако, наблюдается только в направленни $\vec{k}_{4}=2 \vec{k}_{2}-\vec{k}_{1}$. Таким образом, появляется возможность определения времени фазовой релаксации $T_{2}$ по зависимости интенсивности самодифракции от $t_{12}$, что позволяет упростить схему эксперимента.

Во-вторых, угол между направлениями возбуждающих сигнал РССР импульсов должен быть достаточно малым, чтобы трехмерность образованной спектральной решетки не привела бы к исчезновению сигнала. Как показано в $\left[{ }^{13,14}\right]$, условие синхронизма, хорошо известное для трехмерных пространственных голографических решеток, допускающее рассеяние только тогда, когда $\vec{k}_{3}=\vec{k}_{1}$, т. е. в направлении $\vec{k}_{4}=\vec{k}_{2}$, распространяется и на трехмерные спектральные решетки.

В-третьих, применение методики РССР к системам, склонным к фотовыжиганию провалов, требует тщательной проверки и специальных мер предосторожности для исключения влияния процесса выжигания стабильных спектральных провалов в ходе регистрации зависимости сигнала РССР от $t_{12}$.

Кроме того, следует учесть процессы насыщсния, если интенсивности возбуждения настолько высоки, что приводят к значительному изменению заселенности основного уровня.

\section{Схема эксперимента}

В наших экспериментах возбуждение осуществлялось лазером на красителе (ЛК), который накачивался эксимерным лазером на ХеСl (рис. 2). Импульсы ЛҚ направлялись в интерферометр типа Майкел- 
сона, где формировалась пара импульсов с волновыми векторами $\vec{k}_{1}$ и $\vec{k}_{2}$, которые направлялись на объект под углом $2^{\circ}$ относительно друг друга и индуцировали там переходную синусоидальную решетку пропускания в полосе примесного поглощения. Задержка между импульсами изменялась при помощи регулируемой линии задержки.

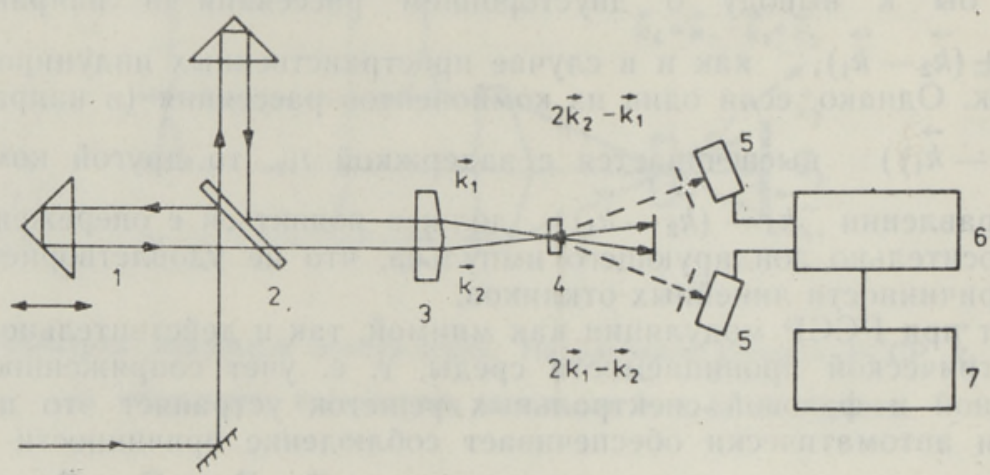

Рис. 2. Схема эксперимента РССР. 1 - линия задержки, 2 - светоделитель, 3 призма френеля, - объект в криостате, 5 - фотодиод, 6 - стробирующий усреднитель, 7 - регистрирующее устройство.

Сигнал самодифракции на динамической спектральной решетке в направлении $2 \vec{k}_{2}-\vec{k}_{1}$ выделялся пространственно и падал на фотодиод. Интенсивность сигнала самодифракции в зависимости от задержки $t_{12}$ регистрировалась при помощи стробирующего усилителя в памяти ЭВМ. Спектральная ширина импульсов ЛК (частота повторения 10 Гц) на длине волны возбуждения $\lambda=619$ нм была 2,4 нм. Этому соответствует ширина пика автокорреляционной функции импульсов возбуждения $\tau_{c} \approx 0,45$ пс, которая определяет временное раз-

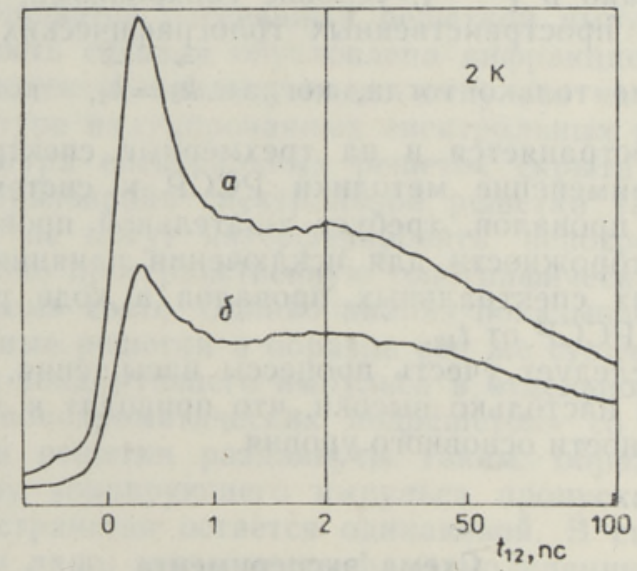

Рис. 3. Зависимость интенсивности сигнала РССР от задержки импульсов $t_{12}$ в образце ОЭП-ПС. Скорость сканирования задержки $t_{12}$ увеличивалась в 33 раза в точке 2,0 пс. Интенсивности возбуждения: $a-I=3 I_{s}, \sigma-I=1,5 I_{s}$, где $I_{s}-$ интенсивность насыщения для бесфононной линии. 
решение в эксперименте. Пиковая интенсивность возбуждения на образце была $50-15$ кВт/см², что обусловливало суммарную дозу облучения до 15 Дж/см² за время регистрации кривой затухания. Объектом исследования служила полистиролевая пленка с примесью октаэтилпорфина (ОЭП-ПС) толщиной $\approx 0,5$ мм и оптической плотностью $D=0,6$. Объект погружался в гелиевый криостат, где температура на участке $T<4,2 \mathrm{~K}$ определялась по давлению паров над уровнем гелия и по калиброванному сопротивлению на участке $T>4,2 \mathrm{~K}$.

Хотя доза облучения была сравнительно большой, сигнал, обусловленный фотовыжиганием стабильных спектральных провалов, не превышал уровня шума и не обнаруживался. Это можно объяснить широким спектром лазерного возбуждения и постоянным перемещением интерференционных максимумов решетки по спектру.

Чувствительность регистрирующей аппаратуры не позволяла нам получать детектируемый сигнал при малых уровнях возбуждения, где заселенность уровней изменяется незначительно и эффекты насыщения не приводят к увеличению скорости затухания сигнала РССР.

В наших условиях возбуждения наблюдалась зависимость скорости затухания сигнала РССР от интенсивности. Поэтому $T_{2}$ определялся с учетом параметра насыщения по двум кривым затухания, снятым при разных интенсивностях возбуждения (рис. 3).

\section{Результаты эксперимента и обсуждение $\left[{ }^{19,20}\right]$}

Полученные кривые затухания сигнала PССР (рис. 3) имеют явно выраженный двухэкспоненциальный характер, обусловленный наличием двух различных процессов дефазировки, соответствующих уширению бесфононной линии $\Gamma_{00} \sim 1 / T_{2}$ и образованию фононного крыла в однородном спектре поглощения $\left[{ }^{21}\right]$. Широкому фононному крылу соответствует быстрый компонент затухания (рис. 3), а узкой бесфононной линии - медленный.

Провал в области 1 пс связан с тем, что быстрый компонент затухает быстрее, чем медленный достигает своего максимума.

Соотношение интенсивностей быстрого и медленного компонентов сигнала РССР зависит от фактора Дебая-Валлера и от интенсивности возбуждения, так как интенсивности насыщения поглощения через фононное крыло и бесфононную линию могут быть различными (см. рис. 3).

Если интенсивность насыщения $\left[{ }^{22}\right]$ обозначить через $I_{s}$, время фазовой релаксации через $T_{2}$, интенсивность возбуждения через $I$, то в насыщенной системе время релаксации будет следующим:

$$
T_{2}^{\prime}=T_{2} /\left(1+I / I_{s}\right)^{1 / 2}
$$

Наши экспериментальные результаты были в хорошем соответс:вии с формулой (3).

На рис. 3 хорошо видны зависимости скорости затухания сигнала и соотношения быстрого и медленного компонентов от интенсивности.

В экспериментах максимальная интенсивность возбуждения была $I=3 I_{s}$, где значение $I_{s}$ взято для медленного компонента затухания. Из-за заметного насыщения поглощения и малого угла $\theta$ между направлениями импульсов наблюдались и высшие порядки рассеяния в направлениях $\vec{k}_{4}=\vec{k}_{2}+n\left(\vec{k}_{2}-\vec{k}_{1}\right)$, где $n=1,2,3$.

На рис. 3 четко наблюдается и сдвиг максимума сигнала от нулевой задержки $\left(t_{1:}=0\right)$, при которой возбуждающие импульсы пере- 
крываются, а также зависимость сдвига от времени фазовой релаксации $T_{2}$ (рис. 4$)$.

Такой сдвиг обусловлен ростом сигнала за счет интерференции вкладов от амплитудной и фазовой составляющих пространственноспектральной решетки при увеличении $\left|t_{12}\right|$ в пределах времени когерентности импульса. В пространственно-временной голографии эта интерференция приводит к большим дифракционным эффективностям по сравнению с пространственными амплитудно-фазовыми голограммами $\left[{ }^{13}\right]$. Зависимость сдвига максимума сигнала РССР от $T_{2}$, определяемая конкуренцией рассмотренного механизма роста сигнала с его ослаблением, согласно формуле (1), позволяет по величине сдвига определить значения $T_{2}$, если они одного порядка или меньше времени когерентности возбуждающего света.

C учетом насыщения поглощения по кривым затухания PCCP вычислены времена фазовой релаксации $T_{2}$ возбужденного состояния октаэтилпорфина в полистироле при температурах $T=2,15-1,57 \mathrm{~K}$. Время дефазировки, соответствующее быстрому компоненту затухания $\left(T_{2}=1,4\right.$ пс), не зависело заметно от температуры.

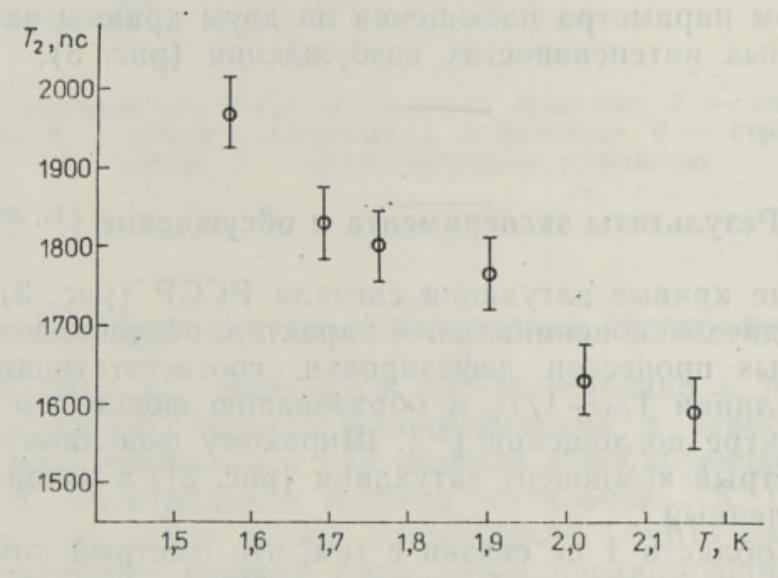

Рис. 4. Температурная завнсимость времени фазовой релаксации $T_{2}$.

Сравнение результатов по фотовыжиганию провалов $\left[{ }^{22}\right]$ (при $T=$ $=1,5-2,0 \mathrm{~K}, T_{2}=0,71-0,49 \mathrm{Hc)}$ указывает на значительный вклад процессов спектральной диффузии в ширину провалов. При $T=$ $=1,5-2,0$ K провалы уширены в $\sim 3$ раза, хотя теория предсказывает для матрицы полистирола более короткие характерные времена спектральной диффузии, чем время измерения в нашем эксперименте. К coжалению, из-за малости температурного промежутка нельзя было достаточно точно определить закон температурного уширения бесфононной линии. По нашим результатам можно лишь утверждать, что $\Gamma(T) \sim T^{n}$, где $0,5<n<0,9$. Кроме того, сравнение с результатами фотовыжигания провалов носит качественный характер, т. к. концентрации примесей в объектах не соответствовали (в нашем объекте концентрация октаэтилпорфина была в $\sim 3$ раза больше і достигала $5 \cdot 10^{-3}$ моль/л). Для получения количественно сравнимых результатов следует провести эксперименты по фотовыжиганию провалов и по PCCP с одинаковыми объектами. 


\section{Заключение}

В данной работе доказано, что методика рассеяния на светонаведенных спектральных решетках с помощью некогерентных лазерных импульсов является плодотворной при изучении сверхбыстрых процессов релаксации в примесных стеклах. Экспериментально установлено наличие процессов спектральной диффузии в твердом растворе октаэтилпорфина в полистироле при $T \sim 2 \mathrm{~K}$ и показана возможность определения времен фазовой релаксации методом РССР в условиях насыщения поглощения.

Авторы выражают благодарность К. К. Ребане за поддержку и постоянный интерес к работе, П. Саари за полезные обсуждения и И. Ренге за приготовление объектов.

\section{ЛИТЕРАТУРА}

1. Гороховский А. А., Каарли Р. К., Ребане Л. А. // Письма в ЖЭТФ, 1974, 20, $474-476$.

2. Kharlamov, B. M., Personov, R. I., Bykovskaya, L. A. // Opt. Commun., 1974, 12, $191-193$.

3. Rebane, L. A., Gorokhovskii, A. A., Kikas, J. V. // Appl. Phys., 1982, B 29, 235-250.

4. Friedrich, J., Haarer, D. // Angew. Chem. (Int. Ed. Engl.), 1984, 23, 113-140.

5. Beach, R., Hartmann, S. R. // Phys. Rev. Lett., 1984, 53, 663-666.

6. Asaka, S., Nakatsuka, H., Fyjiwara, M., Matsuoka, M. // Phys. Rev., 1984, A 29, $2286-2289$.

7. Fujiwara, M., Kuroda, R., Nakatsuka, H. // J. Opt. Soc. Amer., 1985, B 2, 16341639.

8. Defour, M., Keller, J. C., Le Gouët, J. L. // J. Opt. Soc. Amer., 1986. B 3, 544-547.

9. Morita, N., Yajima, T. // Phys. Rev., 1984, A 30, 2525-2536.

10. Golub, J. E., Mossberg, T. W. // J. Opt. Soc. Amer., 1986, B3. 554-559.

11. Hattori, T., Kobayashi, T. // Chem. Phys. Lett., 1987, 133, 230-234.

12. Ребане А. К., Каарли Р. К., Саари П. М. // Письма в ЖЭТФ, 1983, 38, 320-322.

13. Саари П. М., Каарли Р. К., Ребане А. К. // Квант, электроника, 1985, 12, 672-681,

14. Saari, P., Kaarli, R., Rebane, A. // J. Opt. Soc. Amer., 1986, B 3, 527-534.

15. Weiner, A. M., De Silvestri, S., Ippen, E. P. // J. Opt. Soc. Amer., 1985, B 2, $654-662$.

16. Mossberg, T. W., Kachru, R., Hartmann, S. R., Flusberg, A. M. // Phys. Rev., 1979, A $20,1976-1996$.

17. Hesselink, W. H., Wiersma, D. A. // Phys. Rev. Lett., 1979, 43, 1991-1994.

18. Кузнецова Т. Н. // Тр. ФИАН, 1975, 84, 62-164.

19. Каарли P. K., Pятсеп М. Л. Новые методы лазерной спектроскопии молекул в низкотемпературных средах. Таллин, 1987, 39-40.

20. Каарли P. K., Рятсеп M. Л. Люминесценция молекул и кристаллов. Таллин, 1987,61 .

21. Ребане K. K. Элементарная теория колебательной структуры примесных центров

22. Gorokhovskii, A. A., Korrovits, V. N., Palm, V. V., Trummal, M. A. Laser Optics in Condensed Matter. Proc. 3nd USSR-USA Symp. Leningrad, 1987 (in press); Gorokhovskii, A. A., Rebane, $K$. K. Abstracts of the Third International Conference on Unconventional Photoactive Solids. West Germany, Schloss Elman, $1987,75$.

\footnotetext{
Институт физики
Академии наук Эстонской ССР
}

Поступила в редакцию

$1 /$ II 1988 


\section{MADALATEMPERATUURILISTE LISANDISUSTEEMIDE RELAKSATSIOONI- PROTSESSIDE UURIMINE OPTILISELT INDUTSEERITUD SPEKTRAAL- VOREDELT TOIMUVA HAJUMISE ABIL}

On analüüsitud laia spektriga mittekoherentsete laserimpulsside kasutamist lisandisüsteemide ülikiirete relaksatsiooniprotsesside uurimiseks. Kasutades optiliselt indutseeritud spektraalvôredelt valguse hajumise metoodikat on 10-nanosekundiliste laserimpulssidega saavutatud ajaline lahutus 0,45 ps. Eksperimentaalselt on kindlaks tehtud spektraalse difusiooni olemasolu $\mathrm{H}_{2}$-oktaetüülporfiini lisandiga polüstüroolis, milles on leitud faasirelaksatsiooni kiirused temperatuuridel $1,57-2,15 \mathrm{~K}$. Edasi on näidatud, et meetod on kasutatav ka lisandisüsteemi neeldumise tugeva küllastuse korral, ning on uuritud hajumissignaali iseloomu madalatemperatuuriliste lisandisüsteemide korral.

\section{RATSEP and R. KAARLI}

\section{RELAXATION PROCESSES IN LOW-TEMPERATURE IMPURITY GLASS STUDIED BY THE METHOD OF SCATTERING FROM TRANSIENT FREQUENCY GRATINGS}

This paper analyses the method of scattering from a light-induced spectral grating for measuring ultrafast dephasing times in condensed matter. The time resolution $0.45 \mathrm{ps}$ has been obtained by using temporally incoherent nanosecond dye laser pulses with a smooth and broad spectrum. The homogeneous optical dephasing times $\left(T_{2}\right)$ of $\mathrm{H}_{2}$-octaethylporphin in polystyrene glass was measured at temperatures $1.57-2.15 \mathrm{~K}$ and the presence of spectral diffusion has been established. The application of the method in case of high saturation of absorption is considered and the two-exponential character of decay curves is interpreted. 